\title{
Effectiveness of group logo therapy on life expectancy \& loneliness of minority aged community in Southwest Iran
}

\section{Introduction}

The social change is now shaped by the movement of a population wave towards old age. Between 2000 and 2050, the population over the age of 60 will reach from about $11 \%$ to 22 , and the forecast indicates that the absolute number of people aged 60 years old and over rises from 605 million to 2 billion in the period. In Iran, 8.2 percent of the country's population is 60 years old and above. The becoming 25 years, the Iranian older population will be double by the current level. ${ }^{1,2}$ There are most important factors in the incidence and prevalence of mental and physical illnesses associated with aging i.e. depression, suicide, severe disappointment, social isolation and exclusion, impatience, anxiety, discomfort, impairment of physical health problems, nutrition matters, and sleep disorders. ${ }^{2,3}$ Recent literature shows that mental health in the elderly is the result of the interaction between biological, psychological and socio-cultural factors. In other words, mental health in old age is not the result of a change in the passage of time throughout life. ${ }^{3,4}$ As age grows and life passages, the changes such as close relatives, loss of power, life's goals, physical skills, and social status will be occurred regarding old age. The combination of these changes and the approaching of the final days of life and death impose particular pressure on the elderly. The pressure from depression and loneliness is more significant, and both are closely related. ${ }^{5,6}$ Older people living far away from their families are more likely to suffer from loneliness than older people living with their families. Studies have shown that the elderly living near their families are more satisfied with their social network and social connections compared to the elderly living in a nursing home and lonely. ${ }^{7-9}$ One of the important factors affecting the physical and mental health of the elderly social participation, while respecting the rights of the elderly, in maintaining social order and improving the quality of their daily lives can also be useful. ${ }^{10-12}$

In this research the effectiveness of Group Logotherapy (GL) on the increase of life expectancy and the decrease of loneliness has been

Table I Descriptive Statistics of Experiment and Control Groups
Volume 2 Issue 3 - 2017

\author{
Abdolrahim Asadollahi, ${ }^{1,2}$ Farzeneh Bahadori' \\ 'Department of Aging Health \& Promotion, Shiraz University of \\ Medical Sciences, Iran \\ ${ }^{2}$ Australian Centre for Quality of Life, Deakin University, \\ Australia
}

Correspondence: Abdolrahim Asadollahi, Department of Health Aging, School of Health, Shiraz University of Medical Sciences, Iran, Email bahadori_farzaneh@yahoo.com

Received: July 03, 2017 | Published: September 22, 2017

investigated within the retired minority men of aged community in southwest Iran. The subjects (Arab \& Lor Ethnic Minority Groups) were all the retired men who were registered as the member of daily care centers. The sample includes 80 persons who were randomly selected among the 230 aged volunteers in participating of this study. They were divided by two groups: The control and the experimental group. The instruments which were used in this study Miller Scale and revised loneliness scale (UCLA-R). The research design is a semiexperimental design which has a pretest and posttest with control group. The participants received informed consent before participating in the research as well. After randomizing control and experimental groups, the experimental treatment (Group Logotherapy) was done on experimental group for ten session (each session ninety minutes and once a week). After doing the treatment post tests were given to both groups. In order to analyze the data the multivariate analysis of variance (MANOVA) was applied. The result showed that the GL statistically has significant effect to increasing life expectancy and the decrease of loneliness in experimental group in comparison to the control group $(\rho \leq .05)$. Table 1 shows the descriptive characteristics of the control and test groups before and after the examination.

\begin{tabular}{llllllll}
\hline Position & N & Follow up & & Pre-test & \multicolumn{3}{c}{ Post-test } \\
\hline Group & & SD & Mean & SD & Mean & SD & Mean \\
\hline Experiment* & 20 & 5.37 & 33.8 & 7.68 & 34.5 & 7.71 & 42.95 \\
Control* & 20 & 6.88 & 39.1 & 7.47 & 39.55 & 7.68 & 40.35 \\
Experiment ** & 20 & 17.97 & 183.75 & 19.9 & 183.3 & 24.52 & 166.2 \\
Control** & 20 & 19.7 & 175.45 & 20.23 & 175.2 & 20.6 & 173.15 \\
\hline
\end{tabular}

*Feeling Alone

**Life Expectancy

As seen in Table 1, the mean and standard deviations of loneliness scores of retired participants in the test and control group at the pretest and post-test stage indicate that the mean scores of loneliness

in the experimental group Pretest (42.95) was reduced to post-test (34.5), but in the control group, the average loneliness scores did not change significantly ( 40.35 vs. 39.38 ). The mean score of loneliness 
of the experimental group in the follow-up phase is about 0.70 in comparison with the pre-test stage, but the mean of loneliness in the control group does not change compared to the post-test stage (39.55 vs. 39.10). Also, the mean and standard deviations of the life expectancy score of minority retirees in the experimental group in the pre-test and post-test stages indicated an increase in life expectancy of this group (166.20 pretest compared to 183.30 posttest). However, life expectancy at the follow-up stage is not reduced to the post-test stage. It may suggest that the teaching method has retained its effect one month after the intervention. But in the control group, the life expectancy from pre-test to post-test was increased to 2.55 (173.15 versus 175.20), and the mean score of life expectancy in the control group has not too much significantly difference at the follow-up stage with the pre-test and post-test (mean score of follow up: 175.45).

As shown in Table 2, statistical tests of multivariate analysis of variance for the experimental and control groups are significant. These findings indicate that the two groups were significant difference at last in one of the dependent variables. Consequently, the results of ANOVA are shown in Table 3 .

Table 2 Results of MANOVA regarding dependent variables

\begin{tabular}{lllllll}
\hline \multirow{2}{*}{ Tests } & Value & F ratio & Df of error & Df of hypothesis & $\rho$ \\
\hline Group & Pillai's Trace & 0.36 & 5 & 74 & 8 & 0.003 \\
& Wilk's Lambda & 0.63 & 5 & 74 & 8 & 0.003 \\
& Hotelling's T2 & 0.57 & 5 & 74 & 8 & 0.003 \\
& Roy's largest root & 0.57 & 5 & 74 & 8 & 0.003 \\
\hline
\end{tabular}

Table 3 ANOVA Results regarding difference between groups

\begin{tabular}{|c|c|c|c|c|c|c|}
\hline IV & DVs & SS & $\mathbf{F}$ & Mean of SS & DF & $\rho$ \\
\hline \multirow[t]{5}{*}{ Group } & Loneliness (pre and posttest) & 585.22 & 15.74 & 585.22 & I & 0.0001 \\
\hline & Feeling alone (ahead and track) & 624.1 & 20.16 & 624.1 & $\mathrm{I}$ & 0.0001 \\
\hline & Feeling alone (post-test and follow up) & 0.62 & 0.05 & 0.62 & I & 0.824 \\
\hline & Life expectancy loneliness (pre and posttest) & 2265.02 & 7.14 & 2265.02 & I & 0.011 \\
\hline & Life expectancy loneliness (advance and follow up) & 232.62 & 7.23 & 232.62 & I & 0.011 \\
\hline
\end{tabular}

As seen in Table 3, the ratio of observed $\mathrm{F}$, the pre- and post-test difference in the sense of loneliness of the test and control groups is $15.77(\rho<0.0001)$. This finding suggests that the experimental and control groups differ in their sense of loneliness. Also, indicating that the mean score of loneliness in the experimental group was reduced (with a difference of 8.45). This finding confirms the first hypothesis of the research. The ratio of $\mathrm{F}$ analysis of variance related to the pretest and follow-up stage in the loneliness variable is 20.16, which at a significant level of $\rho<0.0001$, indicates that the intervention method in the follow-up section is still effective for the intervention group. Additionally, there is no significant relationship between $\mathrm{F}$ ratio of post-test and follow-up stages $(\mathrm{F}=0.05, \mathrm{SS}=0.62, \rho=0.824)$. This suggests that the educational method has maintained its effect from the post-test to follow-up stage for the intervention group and the positive impact of the teaching method is on the follow-up section.

In addition, the ratio of $\mathrm{F}$ obtained in the intervention and noninterventional groups related to the difference in pre-test and posttest in the life expectancy of life was $7.37(\rho \leq 0.1111)$. This finding suggests that the life expectancy of the experimental and control group varies from pre-test to post-test. The significant difference was in favor of increasing the life expectancy of the experimental group (with a difference of -10.51). The observed $\mathrm{F}$ ratio was related to the pre-test and follow-up of the experimental and the control group in the life expectancy variable was $7.23(\mathrm{p}<0.11)$. This finding suggests that an interventional method for the under-training group shows its effectiveness until the follow-up section. Finally, the observed $\mathrm{F}$ ratio for post-test and follow-up of the experimental and the control group in the life expectancy is statistically not significant $(F=0.01, \rho=0.899)$. That is, the effectiveness of intervention or educational method from the post-intervention section to the follow-up part shows its positive effectiveness.

\section{Conclusion}

We can say that loneliness is an important factor in reducing the general health of the elderly and since the feeling of recognizing loneliness can be effective in maintaining the health of the elderly. Therapeutic meaning extends to the person's field of vision, where meaning and values are seen in the field and his consciousness.

\section{Authors contribution}

FB has contributed to the design, performed the interviews, and wrote the draft. AA have acquisition data, analyzed the data, and interpretation of results and discussion. The both author critically revised and approved the final manuscript.

\section{Acknowledgements}

We wish to thank colleagues, Editor, and the anonymous referees for their valuable comments.

FB has contributed to the design, performed the interviews, and wrote the draft. AA have acquisition data, analyzed the data, and interpretation of results and discussion. The both author critically revised and approved the final manuscript. 


\section{Ethical considerations}

Ethical matters, e.g. plagiarism, uninformed consent, misconduct, data fabrication and/or falsification, double publication and/or submission, redundancy, etc., have been totally observed by the authors.

\section{Patient consent}

Written and verbal consent of patients was obtained before participating in the study.

\section{Authors note}

The opinions expressed by the authors contributing to this article do not necessarily reflect the official position of the SUMS or the institutions with which the authors are affiliated.

\section{Conflict of interest}

The authors declare that they have no competing interests.

\section{References}

1. Najafi GT, Ariapour S, Jafari OM. Epidemiology and Relationship of Fall and Fear of Falling in the Elderly Residing at Kamrani Nursing Home, Tehran, Iran. Salmand. 2016;10(4):152-161.

2. Alaviani M, Khosravan S, Alami A, et al. The Effect of a Multi-Strategy Program on Developing Social Behaviors Based on Pender's Health Promotion Model to Prevent Loneliness of Old Women Referred to Gonabad Urban Health Centers. Int J Community Based Nurs Midwifery. 2015;3(2):132-140.

3. Asadollahi A, Saberi M, Entezari M, et al. Iranian version of 4AT, an instrument for rapid delirium screening for later life. International Journal of Advanced and Applied Sciences. 2016;3(4):33-38.
4. Fakhar F, Navayinezhad S, Foroughan M. The Role of Group Counseling with Logo-Therapeutic Approach on the Mental Health of Older Women. Salmand. 2008;3(1):58-67.

5. Shima A, Hassan T. A Survey on Efficacy of group logotherapy on loneliness and anxiety of death in elderly people. International Journal of Medical Research \& Health Sciences. 2016;5(7):73-77.

6. Lee CH. Effects of Logotherapy with Exercise on Meaning of Life, Ego Integrity and IADL in the Elderly. Taehan Kanho Hakhoe Chi. 2006;36(5):701-709.

7. Moradi S, Fekrazad H, Mousavi MT, et al. The Study of Relationship between Social Participation and Quality of Life of Old People Who Are Member of Senior Association of Tehran City in 2011. Salmand. 2013;7(4):41-46.

8. Lantz J, Lantz J. Meaning, Tragedy and Logotherapy with the Elderly. Journal of Religion \& Aging. 2008;5(4):43-51.

9. Asadollahi A, Rezaei P, Faraji N. Aging Successfully: The Impact of Spirituality on Social Participation in the Process of Living Getting Old. Middle-East Journal of Scientific Research. 2013;15(7):920-925.

10. Asadollahi A, Saberi LF, Faraji N. Validity and reliability of male andropause symptoms self-assessment questionnaire among elderly males in Khuzestan province of Iran. J Midlife Health. 2013;4(4):233-237.

11. Morgan JH. Geriatric Logotherapy: Exploring the Psychotherapeutics of Memory in Treating the Elderly. Psychological Thought. 2012;5(2):2.

12. Moosavi S, Kafi SM, Haghiri M, et al. Comparison of efficiency of cognitive therapy and logo therapy on the depression rate of aged men. International Journal of Psychology and Counselling. 2012;4(11):143-149. 